In 2003, the Honour Society of Nursing, Sigma Theta Tau International and Nursing Spectrum sponsored an "Innovations in Clinical Excellence" contest to recognise exemplars of evidence-based nursing practice. The following 4 papers are winning entries, which are published with permission of the Honour Society of Nursing, Sigma Theta Tau International. A fifth winning paper will be published in the January 2005 issue.

\title{
An evidence-based approach to reducing bed rest in the invasive cardiology patient population
}

\section{PRACTICE PROBLEM}

The most uncomfortable part of hospital admission for patients requiring coronary interventional and/or diagnostic procedures is the time required to lie flat after removal of the indwelling femoral arterial introducer sheath. Conventional practice required a minimum of 6 hours of supine bed rest after sheath removal, often resulting in the problem of back pain.

The Nurse Practitioner/Clinical Nurse Specialist (NP/CNS) for interventional cardiology targeted this problem for further investigation in 1994 and took the lead in determining the process and strategies to be used. A group of interested physicians and nurses was convened, reflecting the multidisciplinary interest in addressing this clinical problem.

\section{TYPE OF EVIDENCE USED}

The basis for the practice of prolonged bed rest was a mix of ritual, research, and expert opinion. The expert consensus was that prolonged bed rest was required to ensure adequate haemostasis at the femoral arterial puncture site. Research, much of it conducted by nurse researchers, had been gradually demonstrating the safety of reducing bed rest times from a high of 24 hours to a low of 6 hours after sheath removal.

\section{METHOD USED TO OBTAIN AND REVIEW EVIDENCE} Electronic databases including Medline and CINAHL were reviewed for all entries matching target search terms. Relevant articles were identified, copies obtained, and the findings were reviewed by the NP/CNS and the medical director of interventional cardiology.

The findings of the literature review revealed a split between patients undergoing diagnostic cardiac catheterisation and percutaneous coronary intervention (PCI) procedures. It was agreed that, initially, the diagnostic and the PCI patients would be treated as 2 distinct groups of patients. Several research studies examined bed rest times $<6$ hours after diagnostic catheterisation, whereas there were no studies published examining bed rest of $<6 \mathrm{hrs}$ after PCI at the time of the review.

\section{PLANNED STRATEGY FOR IMPROVEMENT}

Diagnostic catheterisation group. At a meeting of the interest group in 1996, the results of the literature review were presented. It was decided that the published research supported making a change in practice from 6 hours to 2 hours of bed rest. A plan was developed for introducing the change using close monitoring and follow up for the first 50 inpatients. This would allow for the gradual introduction of the practice change in a controlled fashion.
PCI Group. At this same meeting in 1996, it was decided to explore the possibility of conducting a research study to examine reducing bed rest to $<6$ hours after PCI.

\section{HOW THE STRATEGY WAS IMPLEMENTED}

Diagnostic catheterisation group. The first 50 inpatients were followed up between March and May 1997.

PCI group. A research study proposal was developed and funding successfully applied for and obtained. Nursing staff from a variety of clinical areas were involved in the process of recruitment, data collection, and patient care during the research process. Educational sessions were held to prepare them for their role and to introduce the concept of evidencebased nursing practice.

A total of 354 patients were recruited into the BAC Trial between March 1997 and October of 1998. The start of data collection was timed to coincide with the implementation of the practice change for inpatients undergoing diagnostic cardiac catheterisation. This streamlined the launch and recruitment efforts.

\section{METHOD OF EVALUATION}

Diagnostic catheterisation group. Follow up evaluation of the data from the first 50 patients demonstrated the safety of the practice change and the efficacy in reducing patient discomfort. A decision was made to continue with 2 hours of bed rest for this group.

PCI group. Data analysis verified that 2 hours of bed rest was safe and significantly effective in reducing back pain in patients undergoing PCI. The investigators reviewed the data, and in March 1999, 2 years after data collection began, the findings were presented to the nursing and medical staff. The change in practice was implemented the next day. A process for ongoing evaluation of outcomes was initiated to ensure continued patient safety.

\section{OUTCOMES/RESULTS}

Clinical practice changes. In the end, the diagnostic cardiac catheterisation group and the PCI group, initially divided by the research literature, had the same outcome. Reducing bed rest time from 6 hours to 2 hours after arterial sheath removal and promoting early mobilisation is a safe and effective nursing intervention that prevents and/or reduces back pain, while potentially decreasing costs. Follow up has confirmed the safety and efficacy of this evidence-based change in practice.

Peer communication. The process and findings of our experience were presented by means of 6 oral papers at local, national, and international conferences between 1998 and 2000. A total of 3 poster presentations were made between 
1997 and 1998. Audience members included both nursing and medical professionals.

A summary of the BAC trial results is available on-line in the Registry of Nursing Research, Sigma Theta Tau website (www.nursingsociety.org). Two publications in peer reviewed journals, including one which is available online via Medscape, round out the communication strategy.

Further study. A meta-analysis of the existing research data on bed rest after cardiac diagnostic and interventional procedures is currently under way. Plans after completion of the analysis include conference presentation and submission to the Cochrane Collaboration for possible inclusion in the evidenced-based practice database.

\section{LESSONS LEARNT}

- Identify at least 1 person to champion the project from the beginning. This person must be intimately involved in the process and sustain momentum over time.
- Use all relevant members of the multidisciplinary team. Clinical problems are patient focused, not discipline focused, and therefore usually require the efforts and expertise of different professionals to find appropriate solutions. Excluding other professionals from the process may result in opposition and blocking behaviours when it comes time to implement change.

- Have patience. The process of change takes a long time. It is now 9 years since the bed rest project was initiated and, although change in clinical practice has occurred in our institution, many other centres are still imposing prolonged bed rest on their patients. The meta-analysis currently underway will hopefully continue to add to the body of evidence that other institutions can utilise.

WENDY VLASIC, RN, MScN, CCN(C) Cardiac Care, London Health Sciences Centre London, Ontario, Canada

\section{Sedation management for the adult mechanically ventilated patient}

\section{PROBLEM}

From length of stay (LOS) reports, and as recognised by nurse and physician experts, sedation management was problematic for the mechanically ventilated medical intensive care unit (MICU) patient. The growing body of knowledge about sedation management has not been consistently applied in practice, leading to inadequate or oversedation of mechanically ventilated patients, thus prolonging ventilator hours and MICU and hospital LOS and increasing cost.

The purpose of the evidence-based, sedation management project was to implement evidence-based sedation management practices for the mechanically ventilated critically ill adult patient and thereby, improve patient outcomes related to sedation. Anticipated improvements in patient outcomes were (a) decrease in the duration of mechanical ventilation, (b) decrease in MICU and hospital LOS, and (c) decrease in cost per case. Objectives to achieve these goals include (1) increasing nurses' knowledge regarding evidencebased sedation management practices; (2) increasing documentation of the level of sedation, interventions, and reassessment; and (3) developing and implementing an evidence-based sedation management practice protocol inclusive of analgesic and sedative titration using level of sedation and daily interruption of sedatives for a "wake-up" test.

\section{SYNTHESIS OF EVIDENCE}

Evidence was obtained through advanced search strategies using Medline and CINAHL. MeSH search terms used in the Ovid database searches included conscious sedation, mechanical ventilation, agitation, critical care, critical nursing, drug monitoring, sedatives, narcotics, and Propofol. Two members of the project team reviewed all relevant citations for practice implications. The literature search identified several clinical practice guidelines or protocols for administration of intravenous sedation for mechanically ventilated adults.

The research also corroborates that long term mechanical ventilation is associated with significant patient risks, such as ventilator associated pneumonias, nosocomial infections, debilitation, and the need for a tracheostomy. Research suggests that lack of a sedation protocol in an ICU is associated with prolonged sedation, longer ventilation times, longer hospital stays, and higher costs. Evidence suggests that the use of a protocol directed sedation policy decreases the duration of mechanical ventilation for patients with acute respiratory failure compared with non-protocol directed care. Evidence also indicates that the total duration of continuous intravenous sedation is associated with decreased ventilator hours. The success of a sedation management protocol seems to be achieved in part by allowing rapid clinical decision making at the patient's bedside. Clinical practice guidelines in conjunction with research reports provided the research based evidence to guide practice and achieve the desired clinical outcomes for sedation management in the mechanically ventilated (>96 h) patient.

\section{CHANGE IN PRACTICE}

Changes included development and implementation of an agitation and sedation scale; implementation of a sedation protocol for the adult mechanically ventilated patient, including use of combination drug therapy; and the addition of a daily wake-up assessment. Changes in practice were done through a team approach led by an advanced practice and staff nurse partnership and supported through a department of nursing internship programme.

\section{IMPLEMENTATION STRATEGIES}

Successful evidence-based clinical practice change involves organisational and individual commitment. A sedation management team was formed and consisted of an ICU staff nurse, advance practice nurse, nurse manager, assistant nurse manager, pulmonary fellow, staff physician, and pharmacist. Research evidence, clinical practice guidelines, and protocols were synthesised and shared with physicians and pharmacists to gain their support and feedback. A core group of nurse change champions were provided advanced consultation and education, and pre-printed order sheets were developed to facilitate infusion of the practice change. ICU nurses were educated through posters, verbal inservices, and formal didactic and consultative sessions. Pre-implementation and post-implementation data were analysed, and 
102

audit feedback was used to share results with staff and for reinfusion of the protocol after 6 months.

\section{METHOD OF EVALUATION}

Baseline and post-implementation data were obtained from chart audits, a nursing knowledge survey (60\% return rate), and our Clinical Outcomes and Resource Management department. Descriptive statistics and process control measures were used to analyse the data. Process and outcome indicators were used to evaluate the change in practice. Process indicators included an increased knowledge level of nurses regarding evidence-based sedation management practices (knowledge survey); use of a standard tool to document level of sedation, number of times sedation level was documented per each 24 hours of mechanical ventilation, and routine use of an agitation scale; and daily assessment of the patient without sedation (chart audits). Outcome indicators included length of time on the mechanical ventilator, MICU and hospital LOS of the mechanically ventilated patients (ICD-9 procedure code 9672), and mean hospital cost.

\section{OUTCOMES/RESULTS}

The knowledge survey indicated that staff was knowledgeable about pharmacological aspects of benzodiazepine use, but they were less satisfied with sedation management in general. Data from chart audits indicated that there were no structured mechanisms or tools being used to document the level of sedation (ie, there were 175 different terms being used to document sedation level). After implementation of the agitation and sedation scale and the sedation management protocol, the number of times sedation level was documented increased from 3.5 to 10.9 times per 24 hour period.

After implementation of the sedation management protocol, the average ventilator days decreased from 10.3 to 8.1 (2.2 days). LOS for patients ventilated longer than 96 hours in the ICU decreased from 11.4 to 9.1 (2.3 days). The average hospital cost decreased from approximately $\$ 42000$ to $\$ 26000$, a savings of around $\$ 16000$ per patient or a total institutional cost savings of $\$ 352000$ over a 5 month period.

\section{LESSONS LEARNT}

Change involves both organisational and individual commitment and team effort. This was largely a nurse driven practice change necessitating garnering support and enthusiasm from both physicians and pharmacy. It was necessary to find a key physician to partner with us. Infusion into practice is an ongoing effort in which continued support from nurses, physicians, and pharmacy is difficult. There is an ongoing need to enhance sedation management support and resources, measure the effectiveness of the sedation management protocol, and develop additional evidence-based practice improvements. The project is ongoing in 1 unit and will expand to other adult ICUs to enhance multidisciplinary collaboration and improve patient outcomes.

JOSEPH GREINER, RN, MSN JANE A GREINER, RN, BSN Department of Nursing, University of Iowa Hospitals and Clinics Iowa City, Iowa, USA

\section{You can make a difference in 5 minutes}

\section{BACKGROUND}

During the fall of 1999, an article appeared in the American Journal of Critical Care highlighting the importance of oral care. Using this article as a catalyst, the intensive care unit (ICU) clinical practice council conducted a detailed literature search. The findings of this search were compared with current practice, and opportunities for improvement were identified

Studies have documented that patients in medical ICUs have poorer oral hygiene than non-hospitalised patients. This lack of oral hygiene contributes to the development of oral colonisation. Additionally, oral bacteria aspirated into the lung may result in aspiration pneumonia. Specifically, potential pathogens for ventilator associated pneumonia (VAP) are noted to be present in oral secretions in $67 \%$ of patients orally intubated for $\geqslant 24$ hours. Also, after 24 hours, most suction equipment is colonised with many of the same pathogens cultured from secretions.

Pneumonia is the second most common hospital associated infection and the leading cause of nosocomial death. Healthcare associated pneumonia increases a patient's length of stay in the ICU by 6.1 days and hospitalisation by 10.5 days. This increase in length of stay and need for empiric antimicrobial treatment significantly impacts costs. The primary cause of healthcare associated pneumonia is mechanical ventilation. The estimated average costs associated with ventilator associated pneumonia are \$29369.

\section{ORAL CARE POLICY/PROCEDURE}

Appropriate oral care may be a preventative measure against acquiring VAP. A review of current practice demonstrated that foam swabs were the oral care tool of choice, and the frequency and method of oral care varied. The need for a comprehensive oral care procedure was identified.

A critical care clinical nurse specialist and an ICU registered nurse revised the hospital's oral care policy and procedure. Current research and literature were used to develop this policy. To change practice, all staff were educated on the new policy and procedure, and the following points were communicated. Plaque allowed to accumulate along and below the gingival margin is reported to cause tissue changes within 2-4 days. Brushing a patient's teeth is needed to prevent the formation of plaque, which can be a reservoir for pathogens. A soft suction toothbrush should be used minimally twice a day. Oral care is performed every 2-4 hours. Foam swabs can be utilised between brushings; they are effective for stimulation of mucosal tissues but are minimally effective in removing plaque. The antiseptic oral rinse is a $1.5 \%$ hydrogen peroxide mouth rinse for oral cleansing and reduction of respiratory pathogens. A dedicated oral suction line and covered tonsil suction device are required. The subglottic suction catheter is a single use catheter allowing removal of secretions above the cuff. No other change in practice related to preventing healthcare associated pneumonia was implemented at this time. 


\section{IMPLEMENTATION}

A major barrier to performing proper oral care was the lack of appropriate equipment. The staff worked with a manufacturing company to improve and develop oral care products.

Improving oral care for mechanically ventilated patients was expected to improve the patient's oral health and comfort and decrease colonisation of respiratory pathogens. Measures were selected to evaluate compliance with the new oral care procedure and its impact on patient outcomes. $100 \%$ of mechanically ventilated patients in the ICU were included. The following measurements were utilised: (1) VAP rate, (2) frequency of providing oral care, (3) actual product use, and (4) product evaluation.

\section{RESULTS}

The first indicator measures the rate of VAP. Diagnosis was based on National Nosocomial Infections Surveillance System (NNIS) criteria. Baseline data for the previous 24 months were placed on a u-control chart and demonstrated common cause variation. Therefore, the process was in statistical control. The VAP rate during the baseline period was 5.6 VAPs per 1000 ventilator days. After implementation of the new oral care policy and procedure, the rate dropped to 2.0 VAPs per 1000 ventilator days. Comparative mean reference rates from the NNIS database were 9.9 and 8.7. 33 months of post-implementation data were placed on the u-control chart and demonstrate a positive shift in the process.

Also measured was the frequency of oral care preimplementation and post-implementation. $71 \%$ of the nursing staff participated in this data collection. Prior to implementation, $60 \%$ of nurses reported that oral care was provided $\geqslant 4$ times per shift. After implementation of the new oral care policy and procedure, $93 \%$ of nurses provided oral care $\geqslant 4$ times per shift. The use of a toothbrush also correspondingly increased.

During the first 11 months of 2001, actual product usage was measured and compared with the projected product use per ventilator day. Actual oral care product use was $91 \%$ of

predicted product use. This confirmed that the frequency of oral care and toothbrush use for the mechanically ventilated population had increased. The goal of changing practice was achieved.

The final indicator was to measure nursing staff's satisfaction with the new oral care products. $76 \%$ of the ICU staff completed the survey. 94\% indicated that the oral care system is easy to assemble, easy to use, efficient, and allows complete oral cleansing. 83\% agree that this system saves time. The overall results indicate staff's satisfaction with the redesigned oral care products.

\section{CONCLUSION}

After publication of the above study in the Advocate Journal of Health Care (Spring/Summer 2002), professional journals showed a repeated interest in oral care. Additional articles regarding the Advocate, Good Shepherd Hospital study are found in Geriatric Nursing (2002), RT The Journal for Respiratory Care Practitioners, and Hospitalist \& Inpatient Management Report. Publications occurring in various medical journals targeting different audiences proved how important this patient outcome was to many disciplines.

Throughout this process, the following question must be asked: how is evidenced-based practice finalised? Currently, the Advocate, Good Shepherd Hospital study and several other research based outcome studies have been submitted to the Centers for Disease Control for consideration into their "Guidelines for Health-care Associated Pneumonia". Submission to these guidelines would solidify the importance of oral care. Clinical excellence, however, can only be measured when care is actually performed, and positive patient outcomes are obtained.

BONNIE J. SCHLEDER, RN, MS, CCRN
Intensive Care Unit
LORI PINZON, RN
Quality Improvement
Advocate, Good Shepherd Hospital
Barrington, Illinois, USA

\section{Music intervention in the intensive care unit: a complementary therapy to improve patient outcomes}

\section{PRACTICE PROBLEM}

Inherent in illness and hospitalisation are many stressors, which manifest themselves in patient responses including physiological signs of sympathetic nervous system activation and psychological distress that necessitates treatment with pharmacological agents. A wide range of medical conditions combined with exposure to the unique environmental circumstances of the intensive care unit elicit problematic patient behaviour patterns requiring the application of physical and/or use of chemical restraints. Patient responses to stress experienced as a result of illness and hospitalisation may have deleterious effects, potentially exacerbating pathological conditions and increasing the complexity and decreasing the cost effectiveness of nursing care.

\section{TYPE OF EVIDENCE USED}

Evidence of the beneficial effects of music on the physiological, psychological, and social reaction of the patient was derived from an extensive literature review. Historical sources provided observations relating to the calming and healing effects of music from such diverse sources as Pythagoras, biblical commentary, and treatises from students of the 19th century Philadelphia physician Benjamin Rush. The notes of Florence Nightingale reveal the early interest of nurses in music as a beneficial intervention. Music therapy, which emerged as a distinct discipline in the 19th century, provides a rich source of evidence for music as an auxiliary treatment for the reduction of stress, pain, and anxiety. Medical and nursing literature provided many research and observational studies confirming the belief that music intervention is an appropriate adjunct for relief of symptoms that interfere with the healing process. Research has found that heart rate, respiratory rate, and anxiety are reduced after music sessions in mechanically ventilated patients. Studies demonstrate that music reduces agitation in confused patients, improves mood, and facilitates communication. Research supports the use of music as a pain reduction tool both in cancer and 
104

post-surgical patients. In one study of patients with chronic obstructive airway disease, dyspnoea and anxiety scores showed a significant decrease after music intervention. Procedure related anxiety also demonstrated improvement with music intervention according to a study of patients undergoing flexible sigmoidoscopy. Other articles described the benefits derived from using music therapy either to benefit neonates or to reduce non-adaptive behaviours in patients with Alzheimer's disease.

METHOD USED TO OBTAIN AND REVIEW EVIDENCE Information obtained from a systematic review of $>50$ relevant research articles was synthesised into a computer database and critically analysed. Communication was initiated with other evidence-based resources, such as hospital facilities utilising music for therapeutic purposes. Queries regarding pre-existing clinical standards, along with programme design, development, implementation, evaluation, and the ultimate efficacy of their programme were initiated.

\section{PLANNED STRATEGY FOR IMPROVEMENT}

The strategy planned to achieve improvement of patient wellbeing made the documented benefits of music intervention available to all patients in the intensive care unit.

\section{HOW THE STRATEGY WAS IMPLEMENTED}

Personal compact disc players, speakers, or headphones, and a wide selection of compact discs representing the broad age spectrum and rich ethnic and cultural diversity of the surrounding community were made available for all patients to use in the intensive care unit.

\section{METHOD OF EVALUATION}

A quality improvement pilot study was designed to look at select patient parameters, which then was used to evaluate the efficacy of the music intervention project. Blood pressure, heart rate, respiratory rate, sedation and pain scale scores, medication administration, and the presence of physical restraints were recorded on a data collection sheet before and after the music intervention session. Initial data were obtained within 30 minutes prior to initiating a music intervention session and collected within 30 minutes after the completion of a music session. Demographic data, length of stay, and patient satisfaction scores, when available, were also recorded on the data collection form. Information was entered into a computer database, collated, and analysed for parameter outcomes.

\section{OUTCOMES/RESULTS}

Data analysed from the music intervention quality improvement pilot study of 44 patients correlate with findings in the research literature. Of the 44 patients studied, 23 demonstrated a decrease in heart rate after a music session. Respiratory rates in 24 of the patients studied decreased after a music session. Of the 13 patients able to rate their pain, $23 \%$ of those studied reported a decrease in pain after listening to music. Sedation scale scores for $16 \%$ of patients in the study demonstrated movement toward the midpoint rating of 3, which describes the patient as calm and cooperative. Of 8 patients in physical restraints upon initiation of music, 2 had their restraints removed after administration of music. Patient and family anecdotes demonstrated widespread satisfaction with the music intervention project.

\section{LESSONS LEARNT}

Utilising a best practice model that incorporates organisational and continual quality monitoring of the music intervention project established a framework that successfully translated evidence into practice. Through the use of music intervention, staff nurses were able to creatively address problems and initiate change in daily clinical practice within the hospital setting by implementing evidence-based quality improvement strategies.

MARY KATHLEEN WILKINS, RN, BA, BSN MARGERY L MOORE, RN, BSN Intensive Care Unit, Portland Providence Medical Center Portland, Oregon, USA 